\title{
Das neue Wahlgesetz zur Bundestagswahl 2013. Eine Reform der Reform der Reform ist unvermeidlich
}

\author{
Niels Dehmel und Eckhard Jesse
}

Die Bundestagswahl 2013 wird nach einem neuen Wahlrecht abgehalten. Darauf einigten sich die Fraktionen von CDU/CSU, SPD, FDP und Bündnis 90/Die Grünen im Oktober 2012. In einer ersten Lesung im Deutschen Bundestag am 14. Dezember 2012 erörterten die Parteien die Eckdaten des neuen Gesetzes. Bei der Expertenanhörung im Innenausschuss am 14. Januar 2013 befanden die Sachverständigen die Regelungen für verfassungsgemäß. Der Bundestag verabschiedete das Wahlgesetz am 21. Februar 2013 gegen die Stimmen der Fraktion Die Linke. Wenngleich das neue Bundeswahlgesetz verfassungsgemäß ist, weist es doch weiterhin Schwächen auf: Die Zahl der Abgeordneten dürfte sich erhöhen. Überhangmandate - das eigentliche Übel des Wahlsystems - fallen weiterhin an, werden aber kompensiert. Zudem sind die Regelungen komplizierter geworden und nicht transparent genug. Das neue Bundeswahlgesetz ist insofern nur eine Übergangslösung, eine verständliche und vor allem zukunftsfähige Grundlage steht aus. Eine angemessene Alternative wäre die Rückkehr zum Einstimmensystem von 1949.

\section{Das Bundesverfassungsgerichtsurteil vom 3. Juli 2008 und das Wahlgesetz} vom 29. September 2011

Das Bundesverfassungsgericht hatte in seiner Entscheidung am 3. Juli 2008 einen Teil des Bundeswahlgesetzes wegen des paradoxen Effekts des so genannten negativen Stimmgewichts oder inversen Erfolgswerts ${ }^{1}$ für verfassungswidrig befunden. Inverse Erfolgswerte entstehen beim Zusammenwirken der Überhangmandate mit dem System der verbundenen Landeslisten. ${ }^{2}$ Der Effekt tritt im Rahmen der Unterverteilung auf. Das Aufkommen von Überhangmandaten führt nicht zwangsläufig zu inversen Erfolgswerten, die Wahrscheinlichkeit ihres Auftretens steigt jedoch mit der Anzahl der Überhangmandate. ${ }^{3}$ Ausgangspunkt ist stets der Gewinn von mehr Direktmandaten durch eine Partei in einem Bundesland, als ihr nach Zweitstimmen an Listenmandaten in diesem Land zustehen. Diese überzähligen Direktmandate bleiben der Partei als Überhangmandate erhalten. Bekommt die Partei in diesem Land nun mehr Zweitstimmen und somit ein weiteres Listenmandat - auf Kosten einer anderen Landesliste -, wird dies mit einem Überhangmandat verrechnet. Die Mandatszahl in dem Bundesland bleibt konstant; die Zahl ihrer bundesweiten Manda-

1 Der Frankfurter Staatsrechtler Hans Meyer beschrieb sarkastisch den Effekt 1994. Vgl. Hans Meyer, Der Überhang und anderes Unterhaltsames aus Anlass der Bundestagswahl 1994, in: KritV, 77. Jg. (1994), S. $312-362$.

2 Vgl. Ernst Renatus Zivier, Risiken und Nebenwirkungen: zum Urteil des Bundesverfassungsgerichts vom 3. Juli 2008 („negatives Stimmgewicht“), in: Recht und Politik, 45. Jg. (2009), S. 41 - 48, S. 42.

3 Vgl. BVerfG, Urteil vom 3. Juli 2008. 2 BvC 1/07. 2 BvC 7/07, Abs. 40. Das zweistufige Verrechnungsverfahren bringt zwei Arten von Überhangmandaten hervor: interne und externe. Bei externen Überhangmandaten fallen keine negativen Stimmgewichte an. 
te verringert sich (bei Stimmengewinnen) um eins. Umgekehrt wirkt der Effekt ebenso: Eine Partei kann durch weniger Zweitstimmen in einem Bundesland mit Überhangmandaten ein weiteres Mandat in einem anderen Bundesland hinzugewinnen. Die Mandatszahl im „Überhangland“ bleibt konstant; die Zahl ihrer bundesweiten Mandate erhöht sich (bei Stimmenverlusten) um eins. Ein Zuwachs an Zweitstimmen kann zu einem Sitzverlust, ein Verlust an Zweitstimmen zu einem Sitzgewinn führen.

Das BVerfG hatte den Gesetzgeber aufgefordert, bis zum 30. Juni 2011 eine verfassungskonforme Regelung zu schaffen. ${ }^{4}$ Der Großteil der Parteien nahm die lange Änderungsfrist dankbar zur Kenntnis, blieb - im Gegensatz zur Wissenschaft ${ }^{5}$ - untätig und zeigte keine Absicht, eine baldige Reform anzustreben. Erst am 30. Juni 2011, dem letzten Tag der gesetzten Frist, lagen Entwürfe aller Fraktionen vor. Die Vorschläge der Regierungs- und der einzelnen Oppositionsparteien unterschieden sich grundsätzlich. Einig waren sie sich nur in einem Punkt: Auf weitergehende Reformansätze, die das BVerfG in seinem Urteil angedeutet hatte, verzichteten (mit Ausnahmen der Linken) alle Bundestagsfraktionen. In mehreren Plenardebatten und einer Innenausschussanhörung ließ sich kein parteiübergreifender Kompromiss finden. Bereits im Vorfeld hatte die Regierungskoalition für diesen Fall einen Alleingang angekündigt. Allerdings kamen bei der Sachverständigenanhörung gravierende Mängel an ihrem Gesetzentwurf zur Sprache. Er war unter anderem wegen der Beibehaltung der Überhangmandate von offenkundigen (Macht-)Interessen geprägt, beseitigte den Effekt des inversen Erfolgswerts nicht und schuf mit dem Konstrukt der so genannten Reststimmenverwertung neue Verwerfungen. Die Regierungsfraktionen hielten jedoch an ihrer modifizierten Variante der separaten Sitzzuteilung in den einzelnen Bundesländern fest und legten nach der Innenausschussanhörung am 20. September 2011 lediglich einen neu formulierten Absatz $2 \mathrm{a}$ in $\$ 6$ BWahlG vor. ${ }^{6}$

Drei Monate nach Fristende, am 29. September 2011, beschloss der Bundestag mit der Mehrheit der Regierungskoalition die Novellierung des Bundeswahlgesetzes auf Grundlage des Gesetzentwurfs der Fraktionen von CDU/CSU und FDP in der modifizierten Innenausschussfassung. ${ }^{7}$ Die alternativen Reformentwürfe der Oppositionsfraktionen lehnte das Par-

4 Vgl. die Kritik bei Dieter Nohlen, Erfolgswertgleichheit als fixe Idee oder: Zurück zu Weimar? Zum Urteil des Bundesverfassungsgerichts über das Bundeswahlgesetz vom 3. Juli 2008, in: ZParl, 40. Jg. (2009), H. 1, S. $179-195$.

5 Vgl. Joachim Behnke, Überhangmandate und negatives Stimmgewicht: Zweimannwahlkreise und andere Lösungsvorschläge, in: ZParl, 41. Jg. (2010), H. 2, S. 247 - 260; Franz Urban Pappi 1 Michael Herrmann, Überhangmandate ohne negatives Stimmgewicht: Machbarkeit, Wirkungen, Beurteilung, in: ebenda, S. 260 - 278; Daniel Lübbert, Negative Stimmgewichte bei der Bundestagswahl 2009, in: ebenda, S. 278 - 289. Zur Kritik vgl. Gerd Strohmeier, Die Geister, die Karlsruhe rief - Eine Replik auf die Beiträge zur Wahlsystemreform in Heft 2/2010 der ZParl, in: ZParl, 42. Jg. (2011), H. 1, S. 186 - 193; zur Gegenkritik: Joachim Behnke / Florian Grotz, Das Wahlsystem zwischen normativer Begründung, empirischer Evidenz und politischen Interessen. Ein Kommentar zu Gerd Strohmeier sowie Franz Urban Pappi und Michael Herrmann, in: ZParl, 42. Jg. (2011), H. 2, S. 419 - 425. Siehe umfassend Gerd Strohmeier (Hrsg.), Wahlsystemreform (Sonderband der ZPol), Baden-Baden 2009.

6 Vgl. Gesetzentwurf der Fraktionen der CDU/CSU und FDP, Entwurf eines Neunzehnten Gesetzes zur Änderung des Bundeswahlgesetzes. Drucksache 17/6290 vom 28. Juni 2011; in modifizierter Fassung: Deutscher Bundestag, Beschlussempfehlung und Bericht des Innenausschusses (4. Ausschuss), Drucksache 17/7069 vom 22. September 2011.

7 Vgl. auch den Aufsatz von Christian Hesse in diesem Heft der ZParl. 
lament mehrheitlich ab. ${ }^{8}$ Erstmals wurde die Novellierung des Bundeswahlgesetzes nicht mit einer fraktionsübergreifenden Mehrheit beschlossen, sondern allein von der Regierungskoalition. Nach der Billigung durch den Bundesrat am 14. Oktober 2011 und der Ausfertigung durch den Bundespräsidenten trat das geänderte Wahlrecht am 3. Dezember 2011 in Kraft. ${ }^{9}$ Vertreter der drei Oppositionsfraktionen kündigten bereits in der Bundestagsdebatte am 29. September 2011 an, gegen das Modell der Regierungskoalition vor dem Bundesverfassungsgericht zu klagen. Sie reichten ihren Normenkontrollantrag mit Inkrafttreten des Gesetzes ein. ${ }^{10}$ Die Grünen erhoben ein Organstreitverfahren; 3.063 parteiunabhängige Beschwerdeführer strebten zudem eine Verfassungsbeschwerde an - mit Erfolg. ${ }^{11}$

\section{Das Bundesverfassungsgerichtsurteil vom 25. Juli 2012}

In der mündlichen Verhandlung in Sachen „negatives Stimmgewicht/Überhandmandate“ am 5. Juni 2012 befand das Gericht alle Klagen für zulässig. Der Präsident des BVerfG, Andreas Voßkuhle, kritisierte das Vorgehen der politischen Parteien in der Reformdebatte: „Es wäre Aufgabe der Politik gewesen, rechtzeitig und möglichst einvernehmlich ein neues Wahlgesetz vorzulegen. "12 Beides geschah leider nicht.

Am 25. Juli 2012 verwarfen die Karlsruher Richter das neue Wahlgesetz wegen Verstoßes gegen den Gleichheitsgrundsatz und die im Grundgesetz garantierte Chancengleichheit der Parteien. ${ }^{13}$ In ihrem Urteil kritisierten sie erneut das Handeln der Parteien und beanstandeten drei zentrale Punkte des - in ihren Worten - „ernüchternden“ Bundeswahlgesetzes: den weiterhin auftretenden Effekt des inversen Erfolgswertes im Zusammenhang mit der Verteilung der Bundestagsmandate auf die Bundesländer über die Wählerzahl; das neu eingeführte Konstrukt der Reststimmenverwertung; die Überhangmandate, die beständig in einem Umfang anfallen könnten, „der den Grundcharakter der Verhältniswahl aufhebt“14.

Das BVerfG befand den Ansatz der Regierungskoalition, den Effekt des negativen Stimmgewichts unter Verzicht auf die bundesweite Listenverbindung zu verhindern, grundsätzlich

8 Vgl. Deutscher Bundestag, Stenografischer Bericht. 130. Sitzung vom 29. September 2011, Plenarprotokoll 17/130, S. 15320.

9 Eine Verteidigung der Neuregelung findet sich bei Gerd Strohmeier, Die schlechteste Wahlsystemreform - mit Ausnahme aller anderen, in: Zeitschrift für Politik, 58. Jg. (2011), H. 4, S. 393 - 409. Siehe auch, eher kritisch, Niels Dehmel, Eine (un)glückliche Wahlrechtsreform? Die Debatte über eine notwendige Änderung, in: Eckhard Jesse / Roland Sturm (Hrsg.), „Superwahljahr“ 2011 und die Folgen, Baden-Baden 2012, S. 159 - 171.

10 Vgl. Deutscher Bundestag, a.a.O. (Fn. 8), S. 15298; Hans Meyer, Abstrakte Normenkontrolle von 214 Bundestagsabgeordneten der Fraktionen SPD und Bündnis 90/Die Grünen, 2 BvF 3/11.

11 Vgl. BVerfG, Pressemitteilung Nr. 28/2012 vom 7. Mai 2012; Ute Sacksofsky, Organstreitverfahren der Partei Bündnis 90/Die Grünen, 2 BvE 9/11.

12 Andreas Voßkuble zitiert nach: „Verhandlung über Reform. Zweifel am neuen Wahlrecht“, in: sueddeutsche.de vom 5. Juni 2012, http://www.sueddeutsche.de/politik/verhandlung-ueber-reform-bundesverfassungsgerichthat-zweifel-am-neuen-wahlrecht-1.1374939 (Abruf am 18. Juli 2012).

13 Vgl. Volker M. Haug, Das Bundesverfassungsgericht als Gesetzgeber anstelle des Gesetzgebers. Ein kritischer Blick auf das Wahlrechtrechtsurteil vom 25. Juli 2012, in: ZParl, 43. Jg. (2012), H. 3, S. $658-674$.

14 BVerfG, Urteil vom 25. Juli 2012, 2 BvF 3/11, Abs. 66. 
als „mit dem Grundsatz demokratischer Repräsentation“ 15 vereinbar. Allerdings dürfe dabei nicht die Zahl der tatsächlichen Wähler in einem Bundesland als Maßstab dienen. Die Berechnung der Ländersitzkontingente bewirkte dann inverse Erfolgswerte „nicht nur in seltenen, vernachlässigbaren Ausnahmefällen“16. Der Gesetzgeber habe sich an einer „vor der Stimmabgabe feststehenden Größe wie der Zahl der Bevölkerung oder der Wahlberechtigten “17 zu orientieren. Als Grundlage der Bestimmung der Sitzkontingente müsse eine vom Verhalten der Wahlberechtigten nicht beeinflussbare Größe gewählt werden.

Einen zweiten Verfassungsverstoß stellten für die Richter die Zusatzmandate über die sogenannte Reststimmenverwertung dar. Das Verfahren sollte ursprünglich bewirken, dass Stimmen für kleinere Parteien, die in der Regel keine Direktmandate gewinnen, nicht verloren gehen, sondern bei der Sitzzuteilung im Rahmen einer bundesweiten Verrechnung berücksichtigt werden. Die Vergabe von Zusatzmandaten nach $\$ 6$ Abs. 2a BWG sei jedoch nicht geeignet, dieses Ziel zu erreichen. ${ }^{18}$ Für die Ermittlung der Reststimmenmandate sollten jene Wählerstimmen, die ohne Stimmerfolg geblieben sind, bundesweit summiert und durch die im Bundesdurchschnitt für ein Mandat erforderliche Zweitstimmenzahl dividiert werden. Die zusätzlichen Sitze standen vorrangig jenen Landeslisten einer Partei zu, auf die Überhangmandate entfallen. Sie werden mit diesen verrechnet und nicht zusätzlich vergeben. Anschließend sollte die Vergabe in der Reihenfolge der höchsten „Reststimmen“ erfolgen. Das BVerfG sah in dieser Zuteilung eine ungleiche Behandlung der einzelnen Wählerstimmen und den Eingriff in die Chancengleichheit der Parteien gegeben, „ohne dass dies durch einen besonderen sachlich legitimierten Grund gerechtfertigt wäre" ${ }^{19}$. Verkürzt bedeutete das: Nicht jeder Wähler übt mit seiner Stimme in gleicher Weise Einfluss auf das Wahlergebnis aus.

Als dritten Punkt beanstandeten die Richter die hohe Anzahl von Überhangmandaten, die auch nach dem neuen Wahlrecht auftreten können. Zwar ließen sich diese grundsätzlich mit dem Charakter der personalisierten Verhältniswahl vereinbaren, allerdings hätten sich die politischen Verhältnisse dauerhaft verändert. Ein stetiges Aufkommen von Überhangmandaten in hoher Zahl sei zu erwarten, der Gestaltungsspielraum des Gesetzgebers durch den Grundcharakter der Bundestagswahl als Verhältniswahl aber begrenzt. ${ }^{20}$ Entgegen der Entscheidung vom 10. April 1997, als die Richter die Fünfprozenthürde zur Orientierung benannten, sollte die Zahl der Überhangmandate zukünftig die zulässige Höchstgrenze von etwa 15 Mandaten - einer halben Fraktionsstärke - nicht überschreiten. Obwohl die „Zahl von 15 Überhangmandaten [...] nicht vollständig begründet werden kann“21, wurde sie nach Auffassung des Gerichts dennoch nicht willkürlich gewählt. „Erreichte die Zahl der Überhangmandate Fraktionsstärke, käme ihnen danach ein Gewicht zu, das einer eigenständigen politischen Kraft im Parlament entspräche. "22 Die halbe Fraktionsstärke sei hingegen ein guter Maßstab für die Überhangmandate. Die Beschränkung ist ein Kompromiss zwi-

15 Ebenda, Abs. 71.

16 Ebenda, Abs. 94.

17 Ebenda, Abs. 74.

18 Vgl. ebenda, Abs. 98 - 108.

19 Ebenda, Abs. 101.

20 Vgl. ebenda, Abs. 151.

21 Ebenda, Abs. 144.

22 Ebenda, Abs. 142. 
schen Verhältniswahl einerseits und Direktwahl andererseits. ${ }^{23}$ Dies erklärt jedoch nicht, warum das Gericht Überhänge nicht gänzlich verboten hat, obwohl es „faktisch eine Entscheidung gegen Überhangmandate "24 getroffen hatte. So stellt das Urteil mehr einen Kompromiss zwischen früherer und derzeitiger Rechtsprechung dar, als dass es das eigentliche Problem der Überhangmandate löst und eine einfache Regelung schafft. ${ }^{25}$ Im Gegenteil: Es macht das deutsche Wahlrecht komplizierter. Kritikwürdig war die Festlegung auf die Höchstgrenze von 15 Überhangmandaten. Je acht Überhangmandate für die Union und die SPD wären demnach nicht rechtens, wohl aber 15 für eine Partei.

Erneut oblag es dem Deutschen Bundestag, ein verfassungsgemäßes Wahlgesetz vorzulegen. Angesichts der wenig erquicklichen Vorgeschichte räumte das BVerfG dem Gesetzgeber zu Recht keine weitere Übergangsfrist ein. Das neue Wahlgesetz musste rechtzeitig vor der Bundestagswahl 2013 verabschiedet werden.

\section{Der Gesetzentwurf von CDU/CSU, SPD, FDP, Bündnis 90/Die Grünen vom 11. Dezember 2012 und seine Schwächen}

Die Fraktionen von CDU/CSU, SPD, FDP und Bündnis 90/Die Grünen einigten sich im Oktober 2012 auf die Grundzüge eines Gesetzentwurfs zur Novellierung des Bundestagswahlrechts und brachten ihn am 14. Dezember zu einer ersten Lesung im Parlament ein. ${ }^{26}$ Die Linke legte einen Gegenentwurf vor. Nach einer Expertenanhörung empfahl der Innenausschuss am 20. Februar 2013 dem Plenum die Annahme des Gesetzentwurfs der Fraktionen CDU/CSU, SPD, FDP und Bündnis 90/Die Grünen in einer (leicht) geänderten Fassung. Einen Tag später beschloss der Bundestag die Novellierung des Bundeswahlgesetzes gegen die Stimmen der Fraktion Die Linke. Deren Entwurf wurde abgelehnt. ${ }^{27}$ Der VierFraktionen-Vorschlag verfolgt und erfüllt vier Ziele: die Beseitigung des inversen Erfolgswerts in einem Umfang, den das Verfassungsgericht fordert; die Beseitigung der Überhangmandate in ihrer Wirkung, nicht in ihrem Auftreten; die weitgehende Wahrung des Länderproporzes; das Festhalten an den Grundzügen der personalisierten Verhältniswahl (durch Ausgleichsmandate). ${ }^{28}$

Die Mandate für ein Bundesland sind abhängig von der Bevölkerungszahl. Die Wahlgebiete sind nun voneinander getrennt, Listenverbindungen ausgeschlossen, und die Wählerstimmern werden nicht mehr verrechnet. Inverse Erfolgswerte entfallen in ihrer bisherigen Form. Nach der Ermittlung der Gesamtsitzzahl anhand des Bevölkerungsanteils der Bun-

23 Vgl. Martin Fehndrich, Zum Wahlrechtsurteil des Bundesverfassungsgerichts vom 25. Juli 2012, http://www.wahlrecht.de/news/2012/2012113001.html (Abruf am 18. Januar 2013).

24 Ebenda.

25 Vgl. Volker M. Hang, a.a.O. (Fn. 13).

26 Vgl. Gesetzentwurf der Fraktionen CDU/CSU, FDP, SPD und Bündnis 90/Die Grünen, Entwurf eines Zweiundzwanzigsten Gesetzes zur Änderung des Bundeswahlgesetzes, Drucksache 17/11819 vom 11. Dezember 2012.

27 Vgl. Deutscher Bundestag, Beschlussempfehlung und Bericht des Innenausschusses (4. Ausschuss), Drucksache 17/12417 vom 20. Februar 2013; ders., Stenografischer Bericht. 222. Sitzung vom 21. Februar 2013, Plenarprotokoll 17/222, S. 27590 - 27606.

28 Vgl. ders., Stenografischer Bericht. 215. Sitzung vom 14. Dezember 2012, Plenarprotokoll 17/215, S. $26507-26529$, S. 26514. 
desländer erfolgt die Verteilung auf die jeweiligen Landeslisten gemäß der zu berücksichtigenden Zweitstimmen. Berücksichtigung finden nur Parteien mit einem bundesweiten Anteil von über fünf Prozent. Hierbei wird die Zahl der Zweitstimmen durch einen Zuteilungsdivisor nach dem Sainte-Laguë-Verfahren geteilt. Von der ermittelten Mandatszahl werden schließlich die Direktmandate der Partei abgezogen. Erhält eine Partei mehr Direktmandate als nach Zweitstimmen vorgesehen, verbleiben ihr diese als Überhangmandate. Aus der Berechnung ergibt sich die Mindestsitzzahl, die jeder Partei zustehen muss. In einem zweiten Schritt wird die Gesamtzahl der Bundestagssitze, um Überhangmandate zu vermeiden und Wahlbeteiligungsdifferenzen auszugleichen, so weit erhöht, bis eine Oberverteilung aller Sitze nach Sainte-Laguë an die Parteien zu den jeweiligen Mindestsitzzahlen führt. Diese Zahl bildet die Grundlage für die weitere Berechnung. Wahlrecht.de bezeichnet den ersten Schritt daher zu Recht als „Pseudositzverteilung“29. Es ist lediglich ein notwendiger Rechenschritt. Abschließend wird die neue Gesamtzahl der Bundestagsmandate entsprechend der Zweitstimmen an die Parteien vergeben und nach Sainte-Laguë an die Landeslisten unterverteilt, bis der Zweitstimmenproporz hergestellt ist. ${ }^{30}$

Das neue Sitzzuteilungsverfahren gleicht neben den Überhangmandaten auch die unterschiedlich hohe Wahlbeteiligung in den Bundesländern aus. Zur Ermittlung des Zuteilungsdivisors im ersten Schritt werden die Stimmen in diesem Land durch die ihm zustehenden Sitze geteilt. „Eine geringere Wahlbeteiligung bedeutet daher eine geringere Anzahl an [für den Gewinn eines Mandats benötigten] Zweitstimmen [...]. "31 Ausgleichsmandate kommen folglich denjenigen Parteien zugute, die vorwiegend in Ländern mit hoher Wahlbeteiligung erfolgreich waren. Somit dürfte vor allem Die Linke von der Regelung benachteiligt sein. ${ }^{32}$

Der Gesetzentwurf beseitigt die verfassungswidrigen Bestandteile des Wahlrechts und erfüllt somit die Vorgaben der Karlsruher Richter. Zwar ist der Effekt des inversen Erfolgswerts nicht vollkommen ausgeschlossen (er kann in anderer Form weiterhin auftreten), jedoch hat das BVerfG, wie erwähnt, nicht die Möglichkeit, sondern die Wahrscheinlichkeit seines Auftretens kritisiert. Das Gesetz gleicht auch die Verwerfungen durch Überhangmandate aus. Die Flexibilisierung der Bundestagsgröße wahrt den Zweitstimmenproporz. Ein Anreiz für taktisches Stimmensplitting besteht nicht mehr, da sich die Parteien für einen Vollausgleich der Überhangmandate entschieden haben. Ein Teilausgleich ab dem 16. Überhang, wie vom BVerfG vorgesehen, hätte neue Probleme hervorgerufen. Das Gesetz erhält die Grundzüge des bisherigen Wahlsystems und garantiert, dass „die Personalwahl von Wahlkreisbewerbern nach den Grundsätzen der Mehrheitswahl mit der Verhältniswahl von Landeslisten der Parteien kombiniert ist“"33.

29 Vgl. Martin Fehndrich / Matthias Cantow, Zweiter Versuch nach zweitem Urteil: Wahlgesetzentwürfe eingebracht, http://www.wahlrecht.de/news/2012/2012121301.html (Abruf am 18. Januar 2013).

30 Vgl. ausführlich Gesetzentwurf der Fraktionen CDU/CSU, FDP, SPD und Bündnis 90/Die Grünen, a.a.O. (Fn. 26); Deutscher Bundestag, a.a.O. (Fn. 28).

31 Sebastian Roßner, Neues Wahlrecht zum Bundestag. Es bleibt eine Qual, in: Legal Tribune Online vom 14. Januar 2013, http://www.lto.de/recht/hintergruende/h/neues-wahlrecht-zum-bundestagausgleichsmandate-ueberhangmandate-zweitstimme-wahlen-negatives-stimmgewicht/2/ (Abruf am 18. Januar 2013).

32 Vgl. ebenda.

33 Vgl. Gesetzentwurf der Fraktionen CDU/CSU, FDP, SPD und Bündnis 90/Die Grünen, a.a.O. (Fn. 26), S. 9. 
Das Gesetz ist vom Kompromiss der Parteien geprägt - „zwischen dem, was Karlsruhe vom schwarz-gelben Vorschlag hat stehen lassen, und dem, was die SPD wollte “34. Es war nicht zu erwarten, dass die Akteure ein grundsätzlich neues Wahlrecht schaffen würden: einerseits wegen der kurzen Frist bis zur Bundestagswahl 2013 (die Kandidatenaufstellung in den Wahlkreisen und auf den Landeslisten hatte bereits begonnen; Wahlsystemalternativen, die eine grundlegende Reform oder einen Eingriff in die Listenaufstellung beabsichtigen, entfielen damit von vornherein); andererseits aufgrund der unterschiedlichen parteilichen Interessen (das Bewahren der Überhangmandate bei der CDU/CSU, die Beseitigung der Nachteile durch die Überhänge bei der SPD und die Abkehr von Benachteiligungen einzelner Landeslisten sowie das strikte Festhalten am Zweistimmensystem). Nicht zuletzt ist die Reduzierung möglicher Alternativen ein Ausfluss der mehrfachen Verzögerung der Wahlrechtsreform in den vergangenen Jahren. ${ }^{35}$

So spiegelt der gemeinsame Vorschlag von CDU/CSU, SPD, FDP und Bündnis 90/Die Grünen das Handeln der Parteien in der Wahlrechtsreformdebatte wider: Jeder Akteur verfolgte zunächst seine Interessen. Eigene Zugeständnisse mussten mit Zugeständnissen der anderen einhergehen. Die Suche nach dem „besten“ Wahlrecht wurde zur Suche nach dem „besten“ Wahlrecht für die jeweilige Partei. Im Ergebnis entstand ein Kompromiss, der von „Angeboten“ an jede Gruppe geprägt war. Keine Partei wandte sich in der Wahlrechtsdebatte an die interessierte Öffentlichkeit. Absprachen und Kompromisse kamen vielmehr intern zustande. Die Öffentlichen Anhörungen im Innenausschuss dienten eher der Rechtfertigung und Verteidigung der eigenen Position als der Suche nach einer bestmöglichen Lösung. Im Angesicht der sicheren parlamentarischen Mehrheit bei einer Verabschiedung des Gesetzentwurfs ließen die Fraktionen überwiegend die Bereitschaft zur Behebung offenkundiger Nachteile vermissen. Wahlrechtsfragen blieben auch vier Jahre nach dem ersten Verfassungsgerichtsurteil Machtfragen.

Der komplizierte und in seinen Formulierungen kaum allgemein verständliche Gesetzentwurf ist zwar verfassungsgemäß, weist jedoch entscheidende verfassungspolitische Schwächen auf. Ein wichtiger Kritikpunkt ist die mögliche Aufblähung des Bundestages. Die durch Überhänge und Wahlbeteiligungsdifferenzen entstandenen proportionalen Nachteile für andere Parteien werden durch Ausgleichsmandate kompensiert. Der Bundestag vergrößert sich so. Wie Berechnungen des Bundeswahlleiters zeigen, hätte sich die Zahl der Abgeordneten bei den Bundestagswahlen von 1994 bis 2009 über den ursprünglichen Wert erhöht: moderat 2002 und 2005 um 13 beziehungsweise 15 Sitze, deutlich 1994, 1998, 2009 um 30, 68 und 47 Sitze. $^{36}$

Selbst ohne Überhangmandate ist theoretisch eine Erhöhung der Gesamtsitzzahl möglich. In der Begründung des Gesetzentwurfs heißt es: „Eine Vergrößerung der Zahl der zu vergebenden Sitze kann danach auch dann nötig werden, wenn keine Partei Überhangmandate erzielt hat [...]. ${ }^{37}$ Der Grund: die unterschiedliche Wahlbeteiligung in den Ländern, der

34 Albert Funk, Wahlrecht. Mit Restrisiko und vielleicht nur für den Überhang, in: Der Tagesspiegel vom 14. Januar 2013, http://www.tagesspiegel.de/politik/wahlrecht-mit-restrisiko-und-vielleichtnur-fuer-den-uebergang/7629092.html (Abruf am 18. Januar 2013).

35 Vgl. Martin Fehndrich / Matthias Cantow, a.a.O. (Fn. 29).

36 Vgl. Bundeswahlleiter, Modellrechnung Bundestagswahl 2009: Sitzkontingente mit Ausgleich, 9. Oktober 2012, http://www.wahlrecht.de/doku/doku/20121009.pdf (Abruf am 18. Januar 2013).

37 Vgl. Gesetzentwurf der Fraktionen CDU/CSU, FDP, SPD und Bündnis 90/Die Grünen, a.a.O. (Fn. 26), S. 22. 
differierende Anteil der sonstigen Parteien in den Ländern, die mehrfache Summierung der Rundungsfehler und Differenzen bei der Wahlkreiseinteilung. ${ }^{38}$ Die Wahrscheinlichkeit hierfür ist allerdings gering. Wahrscheinlicher ist eine große Anzahl an Ausgleichsmandaten, die nur auf einer kleinen Anzahl an Überhangmandaten für eine bundesweit kleine Partei beruht. So bewirken Überhangmandate der CSU bundesweit weitaus mehr Zusatzmandate. „Jedes zusätzliche Überhangmandat einer Fünf-Prozent-Partei bedeutet eine Vergrößerung des Bundestages um rund 20 Sitze. " ${ }^{39}$ Eine ähnliche Regel gilt für die beiden großen Parteien: Je schwächer CDU und SPD nach Zweitstimmen abschneiden, desto größer ist die Wahrscheinlichkeit von Überhangmandaten und folglich die Zahl der Ausgleichsmandate. Sarkastisch (und überzogen) drückte es Martin Fehndrich bei der Expertenanhörung im Innenausschuss aus: „Wenn eine Partei mit 30 Prozent der Zweitstimmen fast alle Wahlkreise (in ihrem Wahlgebiet) gewinnt, bedeutet dies eine Vergrößerung des Bundestages um 50 Prozent oder um rund 300 Sitze. “40 Bleibt dies auch nur ein fiktives Szenario, zeigt es doch: Der Bundestag könnte sich „theoretisch bis unendlich“ 41 vergrößern. Die Äußerungen der Parteien im Bundestag, Deutschland habe im Verhältnis zu seiner Bevölkerungszahl eines der kleinsten Parlamente, verfangen demgegenüber nicht. ${ }^{42}$

Die Zuteilung von Ausgleichsmandaten konterkariert zudem in gewisser Weise den Befund, dass Parteien für mehr Stimmen mit einer größeren Mandatszahl „belohnt“ beziehungsweise für weniger Stimmen mit einem Mandatsverlust „bestraft“ werden. Bei der Landtagswahl in Nordrhein-Westfalen 2012 gewann die SPD 99 der 128 Wahlkreise - 23 Mandate mehr als nach Zweistimmen vorgesehen. Diese Überhangmandate zogen insgesamt 33 Ausgleichsmandate für die anderen Parteien nach sich. In der Folge ergab sich ein Kuriosum: Obwohl die CDU mit 26,3 Prozent der Zweitstimmen deutlich verloren hatte, zog sie mit unverminderter Fraktionsstärke in den Landtag ein.

Mit der Vergrößerung des Bundestages sind steigende Kosten verbunden, ferner Kommunikations- und Abstimmungsschwierigkeiten im Parlament. Allerdings stellen Ausgleichsmandate das geringere Übel dar. Überhangmandate, die nicht kompensiert würden, verzerrten das Wählervotum.

Aufgrund der detaillierten Regelungen ist das neue Bundeswahlgesetz äußerst kompliziert geworden. So verfehlt es eine der zentralen Forderungen des Bundesverfassungsgerichts aus dem Jahr 2008, das Wahlrecht „auf eine neue, normenklare, und verständliche Grundlage zu stellen“. Für den Wähler bleibt das Wahlrecht intransparent. Die Partei Bündnis 90/Die Grünen wollte in einem Änderungsantrag zum Gesetzentwurf Defizite beseitigen. ${ }^{43}$ Obwohl er strukturell besser ist, ziehen die Formulierungsänderungen wiederum inhaltliche Probleme nach sich. Eine Vereinfachung des Sitzzuteilungsverfahrens ist nahezu unmöglich. Dies ist einerseits den Spezifikationen und dem Mehrparteienkompromiss geschuldet. Andererseits lässt die bewusste Bewahrung des Systems der personalisierten Verhältniswahl samt seiner Eigenheiten (Zweistimmensystem, Überhangmandate) schwerlich eine einfachere Fassung zu.

38 Vgl. Martin Fehndrich, Stellungnahme anlässlich der öffentlichen Anhörung des Innenausschusses am 14. Januar 2013 in Berlin, Innenausschuss-Drucksache 17(4)634 C, S. 4.

39 Martin Fehndrich / Matthias Cantow, a.a.O. (Fn. 29).

40 Martin Fehndrich, a.a.O. (Fn. 38), S. 2.

41 Ebenda.

42 Vgl. Deutscher Bundestag, a.a.O. (Fn. 28).

43 Vgl. Änderungsantrag der Fraktion Bündnis 90/Die Grünen im Innenausschuss, InnenausschussDrucksache 17(4)625 vom 11. Dezember 2012. 


\section{Möglichkeiten zu einer strukturellen Reform}

Die Wahlrechtsdiskussion seit dem Bundesverfassungsgerichtsurteil vom 3. Juli 2008 war meistens von Vorschlägen geprägt, die einen minimalinvasiven Eingriff vorsahen. Sie bewahrten das Wahlsystem in seinen Grundzügen, ließen die Wahlkreiseinteilung und das Stimmensystem unangetastet. Eine zukünftige Wahlrechtsänderung sollte vor allem strukturelle Änderungen erwägen und normenklare, verständliche sowie transparente Regelungen schaffen. Im folgenden kommen einige Reformvorschläge zur Sprache - allerdings nur solche, die das Verhältniswahlprinzip nicht antasten.

Die wohl einfachste Variante ist eine Reduzierung der Zahl der Wahlkreise und der Listenmandate. Nach Friedrich Pukelsheim führen die zu erwartenden Ergebnisse des Volkszensus 2011 zu einem Neuzuschnitt der Wahlkreise. Er plädiert für ein Herabsetzen der Direktmandate auf 275 und somit für eine Senkung der Ausgangsgröße des Bundestages auf 550 Abgeordnete. Bei einer moderaten Aufblähung würde das Parlament maximal 600 Sitze umfassen und - womöglich dauerhaft - unter der von der Reformkommission 1995 gesetzten Grenze bleiben. ${ }^{44}$ Die neue Wahlkreiskalkulation fiele minimal aus, wenngleich das Verfahren kompliziert ist. Es entstünden weniger und vor allem größere Wahlkreise - langjährige „Erbhöfe“ müssten zusammengelegt und neu verteilt werden. ${ }^{45}$ Das Wahlverfahren und die gesetzlichen Vorgaben blieben bestehen, die Schwächen des neuen Gesetzentwurfs jedoch ebenso: Die tatsächliche Größe des Bundestages ist vor jeder Wahl ungewiss; es ziehen beständig mehr Abgeordnete ein, als einer Partei nach Zweitstimmen Sitze zustehen; Überhangmandate und Ausgleichsmandate bleiben unangetastet.

Einen Schritt weiter ginge eine Veränderung der Mandatsstruktur. Möglich ist eine Reduzierung des Anteils der Direktmandate bei einem Erhalt oder einem Anstieg der Zahl der Listenmandate oder die Erhöhung des Anteils an Listenmandaten bei einer gleichbleibenden Anzahl an Direktmandaten. ${ }^{46}$ Das letztgenannte ist abzulehnen, da die Änderung mit einer deutlichen Erhöhung der Mandatszahl einherginge. Dies widerspricht früheren Entscheidungen und fände nur geringe Akzeptanz. Geeigneter wäre eine Verringerung der Direktmandate von derzeit 50 Prozent auf etwa 40. Die Quote hängt stets von der Fragmentierung des Parteiensystems ab: je zersplitterter, desto größer die erforderliche Reduzierung des Direktmandatsanteils. ${ }^{47}$ Im optimalen Fall wird die Entstehung von Überhangmandaten vollständig verhindert. Die Anpassung der Mandatsstruktur muss einen eventuellen Anstieg der Überhangmandate in Betracht ziehen und gegebenenfalls eine Art „Puffer“ einbauen oder zusätzliche Vorkehrungen treffen, die weiterhin anfallende Überhangmandate neutralisieren. ${ }^{48}$ Die Folge: eine ständige Angleichung an die politischen Verhältnisse. Die Reduzierung

44 Vgl. Friedrich Pukelsheim, Die Zeit der Gesetzlosigkeit ist bald vorbei, in: Berliner Republik, 13. Jg. (2012), H. 6, S. $11-13$.

45 Vgl. Dietmar Hipp / Hauke Janssen, Münchhausen-Check. Muss der Bundestag wirklich aufgebläht werden?, in: Spiegel Online vom 1. November 2011, http://www.spiegel.de/politik/deutschland/ wahlrecht-im-muenchhausen-check-muss-der-bundestag-aufgeblaeht-werden-a-864209.html (Abruf am 8. Februar 2013).

46 Vgl. Daniel Lübbert, Negative Stimmgewichte und die Reform des Bundestags-Wahlrechts, InfoBrief der Wissenschaftlichen Dienste des Deutschen Bundestages, Berlin 2009, S. 20.

47 Vgl. Joachim Behnke, Das Wahlsystem der Bundesrepublik Deutschland. Logik, Technik und Praxis der Verhältniswahl, Baden-Baden 2007, S. 198.

48 Vgl. ders., a.a.O. (Fn. 5), S. 256. 
des Direktmandatsanteils erfordert den aufwendigen Neuzuschnitt sämtlicher Wahlkreise, wodurch sie größer und „unpersönlicher“ werden. Kritiker sehen darin eine Schwächung des Personenwahlelements des deutschen Wahlsystems. Proteste von starken Landesverbänden sind ebenso zu erwarten wie von Parteien mit zahlreichen Direktmandaten. ${ }^{49}$

Ein weiterer Lösungsansatz, der mit einer Veränderung der Mandatsstruktur einhergeht, ist die Einführung von Zweipersonenwahlkreisen. In diesem Fall müssten die Wahlkreise deutlich vergrößert werden. Im Schnitt entsteht aus zwei „alten“ ein „neuer“ Zweimannwahlkreis. Joachim Behnke schlug vor, je zwei Wahlkreise mit aufeinanderfolgenden Wahlkreisnummern zusammenzufassen zwecks Wahrung der Ländergrenzen. ${ }^{50}$ Jeder Wähler hätte weiterhin eine Erst- und eine Zweitstimme. Die Kandidaten mit den beiden besten Erststimmenergebnissen wären direkt gewählt. Es ist möglich, dass eine Partei beide „Wahlkreissieger“ stellt, aber nicht sehr wahrscheinlich. Die stärkste Partei müsste mit ihren beiden Kandidaten doppelt so viele Stimmen erzielen wie der Bewerber der zweitstärksten Partei. ${ }^{51}$ Zudem bedürfte es einer optimalen Aufteilung der Stimmen auf beide Kandidaten, um vor dem Gegner zu „landen“. „,Flächendeckende‘ Gewinne“52 von Direktmandaten durch eine einzige Partei wären nahezu ausgeschlossen. Tatsächlich ginge zumeist ein Mandat an die stärkste und eines an die zweitstärkste Kraft - ob der Abstand zwischen beiden nun knapp oder eher deutlich ist. ${ }^{53}$ Zweimannwahlkreise bewahren die Anzahl der direktgewählten Abgeordneten von 50 Prozent ebenso wie das Zweistimmensystem. Der Vorschlag erhält den Charakter der personalisierten Verhältniswahl; das Verhältnis von Direkt- zu Listenmandaten bleibt identisch. Zweipersonenwahlkreise ermöglichen mehreren (auch kleinen) Parteien als bisher den Gewinn von Direktmandaten. Dieses Modell bedarf einer Ergänzung durch andere Maßnahmen, um Überhangmandate mit Sicherheit zu vermeiden, beispielsweise eine Kompensation durch Ausgleichsmandate. ${ }^{54}$ Die unübersichtliche Etablierung von Zweimannwahlkreisen erfordert den Neuzuschnitt oder zumindest die Angleichung sämtlicher Wahlkreise. Die Vergrößerung der Wahlkreise würde zu Protesten der direktmandatsstarken Landesverbände führen.

Volker von Prittwitz hat ein Einstimmensystem mit implizierter Liste vorgeschlagen. ${ }^{55} \mathrm{Im}$ Unterschied zum herkömmlichen Einstimmensystem treten jeweils zwei Kandidaten jeder Partei zur Wahl an. Das Personenwahlelement erfährt so eine weitere Stärkung. Dem Wähler verbleibt eine Stimme zur Wahl des Kandidaten und seiner Partei. Der Wahlkreissieger zieht weiterhin direkt in den Bundestag ein. Landeslisten existieren im Vorfeld der Wahl nicht. Die 299 Listenplätze besetzen die Kandidaten mit den relativ besten Wahlkreisergebnissen für ihre Partei. „Es ziehen also 299 Wahlkreissieger zusammen mit 299 weiteren vom

49 Gegenwärtig wäre dies vor allem die CDU/CSU, die 2009 in 218 der 299 Wahlkreise den Sieger gestellt hat.

50 Vgl. Joachim Behnke, a.a.O. (Fn. 5), S. 257 f.

51 Vgl. ebenda.

52 Florian Grotz, Abschied von der personalisierten Verhältniswahl? Perspektiven einer Reform des Bundestagswahlsystems, in: Eckhard Jesse / Roland Sturm (Hrsg.), Bilanz der Bundestagswahl 2009. Voraussetzungen, Ergebnisse, Folgen, München 2010, S. 411 - 432, S. 428.

53 Vgl. Joachim Behnke, Überhangmandate bei der Bundestagswahl 2009 - das ewige Menetekel, in: PVS, 51. Jg. (2010), H. 3, S. 531 - 552, S. 549.

54 Vgl. ders., a.a.O. (Fn. 5), S. 256.

55 Vgl. Volker von Prittwitz, Hat Deutschland ein demokratisches Wahlrecht? Von negativen Stimmgewichten zur Personalisierten Verhältniswahl, in: APuZ, B 4/2011, S. 9 - 14. 
Bürger gewählten parteiinternen Siegern in den Bundestag ein. " 56 Die Stimmenanteile der Parteien im Bundesgebiet ergeben sich aus der Summierung der Stimmen der Einzelkandidaten. Für die Ermittlung der Personen, die Mandate erhalten, regt Prittwitz an, für jede Partei eine Vergleichsliste der Stimmenanteile ihrer Wahlkreiskandidaten zu erstellen. Das System vermeidet prinzipiell Überhangmandate und damit inverse Erfolgswerte, da die Wahlergebnisse bundesweit verrechnet werden. Nur in seltenen Fällen wären Überhangmandate in geringer Zahl möglich. Allerdings ist die Prozedur mit der Vergleichsliste wenig transparent. Zudem würde dieses System einen Bruch mit der Tradition bedeuten.

\section{Einstimmensystem als Alternative}

Immerhin liefert der Hinweis auf das Einstimmensystem eine gangbare Möglichkeit für eine Reform des Wahlsystems. Der Wähler sollte eine einzige Stimme haben, die doppelt zählt - zum einen für den Wahlkreiskandidaten, zum anderen für dessen Partei. Das seit 1953 bestehende Zweistimmensystem hat sich nicht bewährt. ${ }^{57}$ Seine Einführung basierte auf zwei Motiven: Zum einen sollten damit Wahlkreisabsprachen zwischen befreundeten Parteien ermöglicht werden, um mit Hilfe der Grundmandatsklausel, die vom Überspringen der Fünfprozentklausel entbindet, kleine Parteien ins Parlament zu hieven. Zum anderen war beabsichtigt, den personellen Effekt zu fördern. Erfolgreiche Wahlkreisabsprachen - die Union stellte keine Wahlkreiskandidaten auf - gab es anfänglich (1953 und 1957) in der Tat, aber sie entsprachen schwerlich demokratischen Intentionen. Hingegen hat die Erststimme niemals eine Funktion als eine Art „Persönlichkeitsstimme“ entfaltet. Die massive Zunahme des Stimmensplitting geht auf Wähler zurück, die mit der Erststimme für den Kandidaten einer großen Partei votieren und mit der Zweistimme für eine kleinere Kraft. Wem die jeweiligen Wähler nahestehen (der großen oder der kleinen Partei), ist nicht mit Sicherheit zu entscheiden.

Bei einem System mit nur einer Stimme kommt die personelle Komponente viel stärker zur Geltung. Wenn nur ein kleiner Teil der Wähler (etwa zwei Prozent) für einen bestimmten Kandidaten der Partei A votiert, obwohl die Partei B die eigentliche Präferenz ist, könnten die Parteien attraktivere Kandidaten präsentieren. Beim herkömmlichen Zweistimmensystem hingegen handelt es sich um eine Scheinpartizipation, denn wenn der Kandidat deutlich mehr Stimmen erhält als seine Partei, so wirkt sich dies unter dem Strich nicht positiv für die Partei aus. Sollte er dadurch den Wahlkreis gewinnen, so zieht sein Gegenkandidat in der Regel über die Liste in das Parlament. Es ist eine Paradoxie: Mit einer Stimme verfügt der Wähler über mehr Einfluss als mit zwei Stimmen. Das ist einer der Fallstricke des Zweistimmensystems.

56 Ders., Personalisierte Verhältniswahl! Reformmodelle des Wahlsystems zum deutschen Bundestag, http://userpage.fu-berlin.de/ -vvp/personalisierte_verhaeltniswahl.htm (Abruf am 18. Januar 2013).

57 Darauf hat schon früh Eckhard Jesse aufmerksam gemacht. Ders., Wahlrecht zwischen Kontinuität und Reform. Eine Analyse der Wahlsystemdiskussion und der Wahlrechtsänderungen in der Bundesrepublik Deutschland 1949-1983, Düsseldorf 1985, S. 261 - 311. 
Das Zweistimmensystem (die erste Stimme ist zweitrangig, die zweite erstrangig) wird von einem beträchtlichen Teil der Wählerschaft nicht begriffen ${ }^{58}$ und provoziert so gravierende Missverständnisse - zum Beispiel dadurch, dass mancher Wähler glaubt, er gehe einen Kompromiss ein, wenn er seine Stimmen splittet. Strategisches Wahlverhalten ist auch bei einem Einstimmensystem möglich, indem etwa jemand, der „sonst“ die FDP wählt, seine Stimme nicht „verschenken“ will und für die CDU votiert oder - gerade anders herum - ein „sonstiger" CDU-Wähler sie der FDP gibt, damit diese in das Parlament einzieht. Hingegen gibt es bei einem Einstimmensystem keinen Zweifel über die Absicht des Wählers. Versuche der Manipulation blieben wirkungslos.

Das Einstimmensystem ist transparent, seine Funktionsweise für den Wähler nachvollziehbar. „Das personifizierte Verhältniswahlrecht bliebe erhalten, aber es gäbe kein Splitting mehr, keine Übergangmandate und auch kein negatives Stimmgewicht. "59 Diese Auffassung ist, was die letzten zwei Punkte betrifft, allerdings nicht richtig. Die Rückkehr zum Einstimmensystem ${ }^{60}$ würde zwar Überhangmandate einschränken, weil dann das Stimmensplitting, eine (nicht die einzige) Ursache für Überhangmandate, wegfiele, aber sie würde sie nicht ausschließen (und auch nicht das negative Stimmgewicht). Es gibt jedoch zwei Möglichkeiten, sie prinzipiell zu vermeiden: zum einen dadurch, dass die in einem Land für eine Partei anfallenden Überhangmandate mit deren Listenmandaten in anderen Ländern verrechnet werden $^{61}$; zum anderen, wie erwähnt, durch eine Verkleinerung der Zahl der Wahlkreise. Sollten Überhangmandate noch immer entstehen (dann „externe“), wären (wenige) Ausgleichsmandate unumgänglich. Sie würden keinesfalls zu einer Aufblähung des Bundestages führen.

Die (nicht ausgeglichenen) Überhangmandate sind das Übel des Wahlverfahrens zum Bundestag gewesen - keineswegs deshalb, weil dadurch Stimmen- und Mandatsanteil voneinander abwichen (das gilt auch für die Fünfprozenthürde); vielmehr ist ihr Auftreten nicht berechenbar, zum Teil zufällig ${ }^{62}$, und aus einer Stimmenminderheit (etwa für ein politisches „Lager") kann eine Mandatsmehrheit hervorgehen. Insofern war die Kompensation der Überhangmandate durch Ausgleichsmandate überfällig.

Gewiss, die kleineren Parteien wären von einer solchen Reform wahrlich nicht begeistert, weil sie von einem Zweistimmensystem offenkundig profitieren. Insofern ist es um die Realisierbarkeit nicht gut bestellt. Dieser Befund kann jedoch keineswegs den Verzicht auf derartige Plädoyers nahelegen. Damit gäbe die Politikwissenschaft ihre Funktion als Orientierungswissenschaft auf.

58 Vgl. Rüdiger Schmitt-Beck, Denn sie wissen nicht, was sie tun ... Zum Verständnis des Verfahrens der Bundestagswahl bei westdeutschen und ostdeutschen Wählern, in: ZParl, 24. Jg. (1993), H. 3, S. $393-415$.

59 Christoph Seils, Schafft die Zweitstimme ab!, in: Cicero Online vom 24. Januar 2013, http://www. cicero.de/berliner-republik/wahlrecht-mathematik-schafft-die-zweitstimme-ab/53237 (Abruf am 25. Januar 2013).

60 Sie wird in letzter Zeit von Teilen der Wissenschaft und der Publizistik propagiert. Vgl. beispielsweise Hans Meyer, Die Zukunft des Bundestagswahlrechts. Zwischen Unverstand, obiter dicta, Interessenkalkül und Verfassungsverstoß, Baden-Baden 2010; Frank Decker, Brauchen wir ein neues Wahlrecht?, in: APuZ, B 4/2011, S. 3 - 9, S. 8; ders., Schafft das Zweistimmensystem ab!, in: Berliner Republik, 13. (2012), H. 5, S. 6 f.; Christoph Seils, a.a.O. (Fn. 59).

61 Damit ließe sich zwar der Regionalproporz nicht mehr gewährleisten, aber das wäre hinnehmbar, denn Parteienproporz ist wichtiger als Länderproporz.

62 Anders Volker M. Hang, a.a.O. (Fn. 13), S. 672 f. 


\section{Perspektiven}

Die Halbwertzeit des neuen Bundeswahlgesetzes wird wesentlich vom Ausgang der Bundestagswahl 2013 abhängen. Entstehen nur relativ wenige Überhang- und damit auch wenige Ausgleichsmandate, deuten das Handeln und die Äußerungen der Parteien darauf hin, dass sie keine weiteren Änderungen am Wahlsystem anstreben. Dies käme einem Trugschluss gleich, wie die exemplarisch angeführten Schwächen zeigen. Werden die Abgeordneten nach einem zwar funktionierenden, aber in seiner Struktur intransparenten und komplizierten Wahlsystem bestimmt, das funktionelle Nachteile mit sich bringt, dürfte dies Verdrossenheit und Unverständnis beim Wähler erhöhen.

Das Ziel einer erneuten Wahlrechtsdiskussion muss ein parteiübergreifender Gesetzentwurf sein, der ein zukunftsfähiges und vor allem klar strukturiertes wie verständliches Wahlrecht hervorbringt. Die Parteien wären gut beraten, bereits vor der Bundestagswahl $2013 \mathrm{zu}$ erklären, dass sie das Wahlsystem in der nächsten Wahlperiode strukturell überarbeiteten werden - unabhängig vom Ausgang der Wahl. Gewiss, das „Ei des Kolumbus“ gibt es nicht. ${ }^{63}$ Eine Lösung, die allen demokratietheoretisch sinnvoll erscheinenden Prinzipien Rechnung trägt (und schon gar nicht eine, die alle politischen Interessen berücksichtigt), ist somit schwerlich möglich; aber dass der Kompromiss des Bundestages auf dem kleinsten gemeinsamen Nenner der Parteien beruht, dürfte nicht der Weisheit letzter Schluss sein.

„Machtinteressen sollten in einer Demokratie genau dann nicht ins Spiel gelangen, wenn es um die Regeln der Machtverteilung selbst geht. "64 Die Wahl ist in einem demokratischen Verfassungsstaat das wichtigste Partizipationsinstrument. Daher sollte das Wahlsystem, das Stimmen in Mandate umsetzt, transparent sein. Das neue Wahlsystem erfüllt dieses zentrale Postulat nicht.

63 Suzanne S. Schüttemeyer spricht von einer „eierlegende[n] Wollmilchsau“. So dies., Editorial, in: ZParl 43. Jg. (2012), H. 3, S. 505 f., S. 505.

64 Joachim Behnke, Ein sparsames länderproporzoptimierendes parteienproporzgewährendes automatisches Mandatszuteilungsverfahren mit Ausgleich ohne negatives Stimmgewicht, in: ZParl, 43. Jg. (2012), H. 3, S. 675 - 693, S. 676. 